\title{
Evidence of effectiveness of a fracture liaison service to reduce the re-fracture rate
}

\author{
A. Nakayama $^{1}$ - G. Major ${ }^{1,2}$ - E. Holliday ${ }^{2,3}$ - J. Attia ${ }^{2,3}$ - N. Bogduk ${ }^{1,2}$
}

Received: 22 June 2015 / Accepted: 25 November 2015 /Published online: 9 December 2015

(C) The Author(s) 2015. This article is published with open access at Springerlink.com

\begin{abstract}
Summary We assessed the ability of a fracture liaison service (FLS) to directly reduce re-fracture risk. Having a FLS is associated with a $\sim 40 \%$ reduction in the 3-year risk of major bone and $\sim 30 \%$ of any bone re-fracture. The number needed to treat to prevent a re-fracture is 20 .

Introduction FLS have been promoted as the most effective interventions for secondary fracture prevention, and while there is evidence of increased rate of investigation and treatment at institutions with a FLS, only a few studies have considered fracture outcomes directly. We therefore sought to evaluate the ability of our FLS to reduce re-fracture risk.

Methods Historical cohort study of all patients $\geq 50$ years presenting over a 6-month period with a minimal trauma fracture (MTF) to the emergency departments of a tertiary hospital with a FLS, and one without a FLS. Baseline characteristics, mortality and MTFs over a 3-year follow-up were recorded. Results Five hundred fifteen patients at the FLS hospital and 416 patients at the non-FLS hospital were studied. Over 3 years, 63/515 (12\%) patients at the FLS hospital and 70/ $416(17 \%)$ at the non-FLS hospital had a MTF. All patients were analysed in an intention-to-treat analysis regardless of whether they were seen in the FLS follow-up clinic. Statistical
\end{abstract}

G. Major

gabor.major@hnehealth.nsw.gov.au

1 Department of Rheumatology, Bone and Joint Centre Royal Newcastle Centre/John Hunter Hospital, Lookout Road, New Lambton Heights, Newcastle, New South Wales 2305, Australia

2 Faculty of Medicine University of Newcastle, Newcastle, New South Wales 2308, Australia

3 Hunter Medical Research Institute, Newcastle, New South Wales 2308, Australia analysis using Cox proportional hazard models in the presence of a competing risk of death from any cause was used. After adjustment for baseline characteristics, there was a 30\% reduction in rate of any re-fracture at the FLS hospital (hazard ratio (HR) 0.67 , confidence interval $(\mathrm{CI}) 0.47-0.95, p$ value 0.025 ) and a $\sim 40 \%$ reduction in major re-fractures (hip, spine, femur, pelvis or humerus) (HR 0.59 , CI $0.39-0.90, p$ value 0.013).

Conclusions We found a $\sim 30 \%$ reduction in any re-fractures and a $\sim 40 \%$ reduction in major re-fractures at the FLS hospital compared with a similar non-FLS hospital. The number of patients needed to treat to prevent one new fracture over 3 years is 20 .

Keywords Bone/prevention and control · Fracture liaison service $\cdot$ Fractures $\cdot$ Osteoporosis/epidemiology Osteoporotic fractures/prevention and control $\cdot$ Secondary prevention

\section{Introduction}

Minimal trauma fracture (MTF) is the cardinal risk of osteoporosis [1-5]. In particular, patients who suffer a first fracture are at greater risk of a second fracture, within 1 or 2 years of the first, if their osteoporosis remains untreated [2]. In the case of hip fractures, almost half have a prior history of a MTF [6]. The impact on the individual is devastating. For example, hip fractures have a $24 \% 12$-month mortality, $85 \%$ of survivors need assistance to walk and $20 \%$ require nursing home care [7-9]. While the age-specific incidence of hip fractures may be declining, the actual numbers are continuing to increase due to the numbers of older persons in the population $[10$, 11]. The steeply rising incidence of MTFs is creating an increasing public health and economic burden. The problem is 
worldwide with projected costs rising to 37 billion Euros in Europe by 2025 and 12.5 billion US dollars in China by 2020, with similar projections for the Americas and the Pacific including Australia [12]. This has led to position papers being issued by the International Osteoporosis Foundation (IOF) and the American Society for Bone and Mineral Research calling for the creation and implementation of fracture liaison services (FLS) as the most efficient way of addressing the problem [12-14]. These services are designed to identify patients who suffer a first fracture, assess their bone metabolic status and institute medical therapy as indicated, in order to prevent a second fracture. There has been a broad acceptance of these proposals, and as a measure of their implementation, the IOF website (www.capturethefracture.org) lists 109 sites worldwide with an active FLS.

The value of FLS and similar services has been supported by a number of published analyses of their effectiveness. These studies have shown increased patient assessment and commencement of treatment with projected clinical benefits and cost savings [15-18]. However, there remains a paucity of studies looking at the effectiveness of FLS in achieving their primary task of reducing re-fracture rates [19-22]. At the same time, recurring criticism of the strategy of focusing on the treatment of osteoporosis as the best way to reduce fragility fractures highlights the importance of evaluating intervention outcomes in terms of their actual impact on the fracture rate [23-25].

Earliest studies either relied on historical data for controls [22] or compared rates of subsequent fracture in patients who did or did not attend a designated clinic within the FLS [20]. The control group was a sample of eligible patients who elected not to attend the clinic, which introduces a risk of sampling bias. Likewise, a later study that compared patients attending and not attending a review clinic used one in four samples, in chronologic order, of patients choosing not to attend the clinic [21]. A third study compared re-fracture rates between all patients presenting either to a hospital with an FLS or one with no such service. Capturing all patients generated large sample sizes and small confidence intervals of proportions. A significant difference in favour of the FLS was only demonstrable however as a time dependant outcome when cumulative fracture incidence over time was calculated [19].

The present study was undertaken to evaluate the effect of a FLS on re-fracture risk and provide grounds for outcome-based decision-making about the value of having such a service. In light of the literature, the study was designed to recruit a large sample of patients attending a hospital with or without a FLS and analyse results on an intention-to-treat basis.

\section{Methods}

\section{Programme description}

John Hunter Hospital is a tertiary public hospital and the major trauma referral centre for the Hunter New England Local Health District (located in Newcastle, New South Wales, Australia). The Hunter New England Local Health District covers a region of $131,785 \mathrm{~km}^{2}$, spanning 25 local government areas with an estimated resident population of 873,741 people; $19.4 \%$ of residents are born overseas and $4 \%$ are Aboriginal and Torres Strait Islander people. The John Hunter Hospital FLS has been in operation since 2007. It is a service which identifies, investigates and treats patients with MTFs. Most patients who are identified as having a MTF are automatically given an appointment at the FLS clinic. For logistical reasons, patients discharged to an Aged Care Facility (nursing home or hostel) and those who live out of the area are not given an automatic appointment. These patients are sent a letter and pamphlet informing them that they have had a MTF and should be investigated and treated, with a recommendation to see their general practitioners. Their general practitioner is also sent a letter including information on a local health website that guides osteoporosis investigation and management. Other patients who are not seen in clinic may be patients who have died, are palliative, have been seen as an inpatient or those who choose not to come to clinic after being given an appointment. All patients seen in the FLS clinic are investigated for osteoporosis and secondary causes of osteoporosis, given lifestyle and dietary education and treated for osteoporosis if appropriate.

The comparator hospital was chosen because it is a tertiary public hospital in NSW of similar size to the John Hunter Hospital, is the major trauma referral centre of its area and does not have a FLS. The non-FLS hospital is an 877-bed hospital (2015), providing state-wide services in areas such as trauma. The local health district spans seven local government areas and covers a region with a resident population of 888,000 people. The area is home to people from diverse cultures with $40 \%$ born overseas, with the most common countries of birth outside Australia being Fiji (3.4\%), Iraq (3.3\%) and Vietnam (2.8\%). The hospital has no specific osteoporosis or secondary fracture prevention service but does have endocrinology, rheumatology and aged care services.

The study was approved by the Hunter New England Human Research Ethics committee.

\section{Data collection}

At both the FLS hospital and the non-FLS hospital, lists were created of patients who were aged $\geq 50$ years, and had had a diagnostic code for fracture from their emergency department (ED) presentation described by S02.0 (Fracture of Skull) to 
S92.9 (Fracture of Foot) but excluding any where the description includes "sprains and strains", "dislocation", "injury", "wound", "trauma" or "burn" between July and December 2010. Patients without a MTF, or those that were seen at the FLS clinic before July 2010, were excluded. A MTF was defined as a fracture from a fall from a standing height or less trauma. This was determined by looking at ambulance, triage, ED clinical notes, discharge summaries or inpatient progress notes that were electronically stored on the respective hospital's computer systems. If patients were diagnosed as having a fracture but their imaging reported no fracture, these patients were also excluded. Given the size and historical nature of the study, only information that could be obtained through the hospital computer systems could be collected. Baseline characteristics that were considered to be potential confounders were collected. These included age, gender and postcode as a surrogate marker of socioeconomic status, aboriginal and Torres Strait Islander status, type of initial baseline fracture, whether patients were on treatment at discharge from hospital, and discharge to an Aged Care Facility (either hostel or nursing home). At the FLS hospital, it was recorded whether the patient received a clinic appointment and whether they attended. The date and type of re-fractures were recorded if they were MTFs and were identified on each hospital computer system by looking through subsequent hospital encounters and radiology reports. Re-fractures could be identified only if patients had re-presented to the same hospital or a hospital that was part of the same computer network. Death was recorded from the hospital computer system and confirmed through the NSW State Death and Birth Registries by matching their names and date of births.

\section{Statistical analysis}

We used Cox proportional hazard models to model the eventspecific risk (cumulative incidence function) of bone refracture during follow-up in the presence of a competing risk using the method of Fine and Gray [26]. The competing risk was defined as death from any cause. This method is a modification of the Cox proportional hazard model that accounts for participants who experience a competing event (death) that prevents observation of the event of interest (bone re-fracture). The modification involves retaining participants in the risk set with a diminishing weight when they die, rather than simply censoring them at the time of death. This is the most widely accepted method for unbiased estimation of incidence rates and exposure effects in the presence of competing risks [27]. Covariate effects (hazard ratios) are modelled in relation to the event-specific sub-distribution and are expressed as subdistribution hazard ratios. We employed intention-to-treat principles to compare the re-fracture rates including all patients with minimal trauma fracture regardless of whether they were seen in the FLS clinic or not.
The covariates included in multivariate models included the following:

1. Patient age at admission for original fracture (as a categorical variable: $<65$ or $\geq 65$ years) The cutoff of 65 years was chosen a priori as a standard threshold for defining a senior citizen/retirement age. Age was not used as a continuous value to avoid the assumption of a linear effect.

2. Patient gender

3. Patient socioeconomic status, approximated by the Australian Bureau of Statistics (ABS) Socio-Economic Index For Area (SEIFA) Index of Relative Socio-Economic Advantage and Disadvantage (IRSAD) [28] for the patient's residential postcode.

4. Skeletal bone sustaining the original fracture. This was categorised as major (hip, spine, femur, pelvis or humerus) or minor (all other bones including radius, ulna, hand, tibia, fibula, foot, ribs, clavicle, facial, patella, scapula, skull and sternum).

Patients' treatment status and Aged Care Facility status was not included in the model as these data were collected on the patient at discharge and therefore may have been influenced by the intervention. A record of medications on admission to ED was not consistently available in ED admission notes, especially if the patient was discharged from ED back to home and not admitted to hospital, and when it was present, it was incompletely recorded. We judged that there was too much missing data for this field to be included in this analysis. Although Aged Care Facility status was not included in the analysis, postcode of residence was captured and used to link to the SEIFA code which was used as a measure of socioeconomic status and adjusted for. The multivariate modelling approach involved including all variables (exposure and covariates) in the initial model. Covariates were then removed if their association with the outcome was non-significant (at $\alpha=$ 0.1 ), and their removal produced no substantive change in coefficients for the remaining variables. A substantive change was defined as greater than approximately $10 \%$. The justification for model reduction was to maximise power, since the number of events was modest for some analyses. For present purposes, only the final reduced model is reported.

The proportional hazard assumption was checked by including each predictor variable as a time-varying covariate and ensuring no significant time variation was observed. If significant time variation was observed for a given predictor variable, the interaction between that variable and analysis time was included in the model as an additional term.

For the final model, results are presented as subdistribution hazard ratios for risk factors at the patient level. Results were considered significant if the $p$ value was $<0.05$. Based on final multivariate models, cumulative incidence functions (also known as cause-specific sub-distributions) 


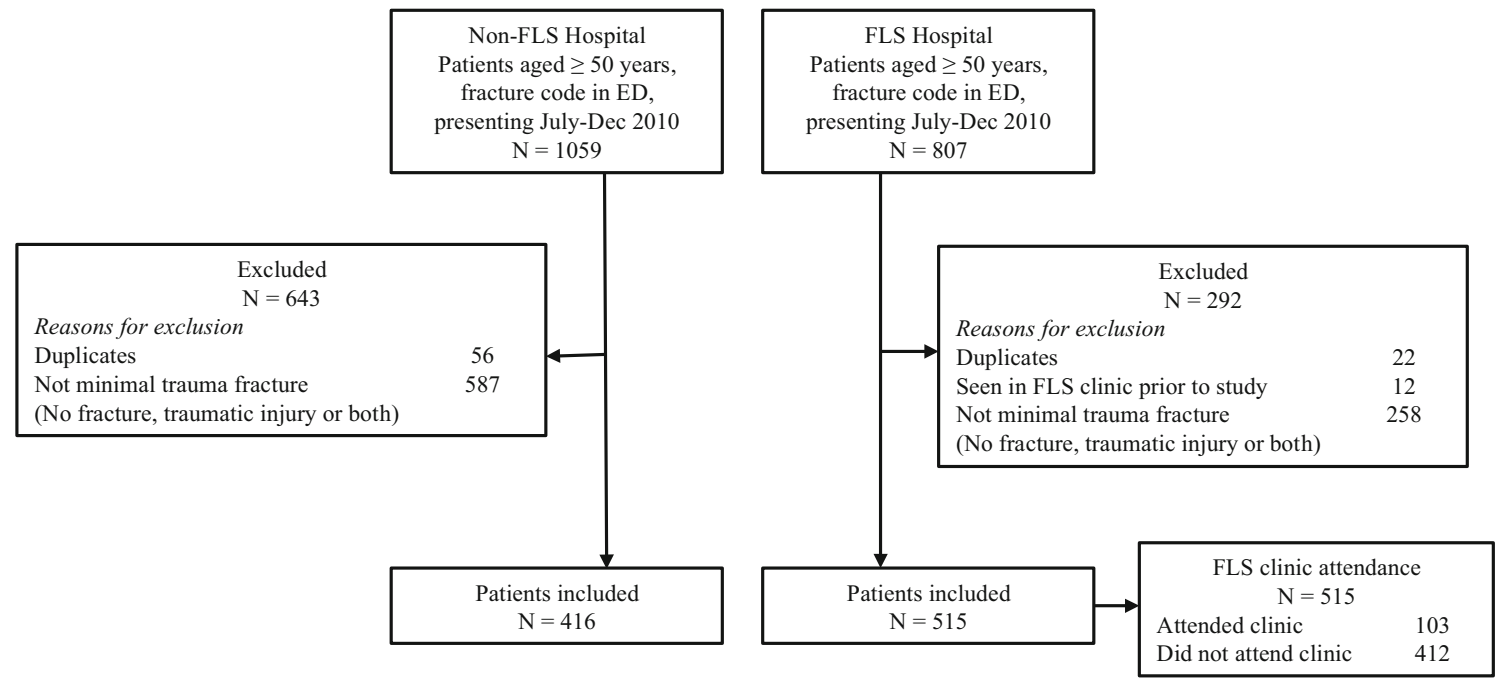

Fig. 1 Flow diagram

were estimated and plotted for the two hospitals (FLS and non-FLS hospital), adjusted for all other covariates retained in the model.

All statistical analyses were programmed using Stata v13.0 (Stata Corp Ltd, College Station, TX).

\section{Results}

The total numbers of patients aged $\geq 50$ years, with a fracture ED code, presenting July-December 2010 were 807 at the FLS hospital and 1059 at the non-FLS hospital. Duplicates,
Table 1 Patient characteristics at the two recruiting hospitals

\begin{tabular}{|c|c|c|c|}
\hline Characteristic & Class/statistic & Non-FLS hospital & FLS hospital \\
\hline Total patients & & $416(44.7 \%)$ & $515(55.3 \%)$ \\
\hline \multirow[t]{2}{*}{ Age } & Mean (SD) & $75.0(12.1)$ & $76.6(12.4)$ \\
\hline & Median (min, max) & $77(50,99)$ & $79(50,100)$ \\
\hline \multirow[t]{2}{*}{ Age group } & $<65$ & $99(23.8 \%)$ & $102(19.8 \%)$ \\
\hline & $\geq 65$ & $317(76.2 \%)$ & $413(80.2 \%)$ \\
\hline \multirow[t]{2}{*}{ Gender } & Male & $110(26.4 \%)$ & $127(24.7 \%)$ \\
\hline & Female & $306(73.6 \%)$ & $388(75.3 \%)$ \\
\hline \multirow[t]{3}{*}{ Fracture type ( 3 categories $)^{\mathrm{a}}$} & Hip & $95(22.9 \%)$ & $199(38.6 \%)$ \\
\hline & Major (excluding hip) ${ }^{\mathrm{b}}$ & $126(30.4 \%)$ & $114(22.1 \%)$ \\
\hline & Minor $^{\mathrm{c}}$ & $194(46.8 \%)$ & $202(39.2 \%)$ \\
\hline \multirow[t]{2}{*}{ Fracture type ( 2 categories $)^{\mathrm{a}}$} & Major $^{\mathrm{b}}$ & $221(53.3 \%)$ & $313(60.8 \%)$ \\
\hline & Minor $^{\mathrm{c}}$ & $194(46.8 \%)$ & $202(39.2 \%)$ \\
\hline \multirow[t]{2}{*}{ Re-fracture during follow-up } & Yes & $70(16.8 \%)$ & $63(12.2 \%)$ \\
\hline & No & $346(83.2 \%)$ & $452(87.8 \%)$ \\
\hline \multirow[t]{3}{*}{ Re-fracture type } & None & $346(83.2 \%)$ & $452(87.8 \%)$ \\
\hline & Major $^{\mathrm{b}}$ & $50(12.0 \%)$ & $42(8.2 \%)$ \\
\hline & Minor $^{\mathrm{c}}$ & $20(4.8 \%)$ & $21(4.1 \%)$ \\
\hline \multirow[t]{4}{*}{ Number of re-fractures } & 0 & $346(83.2 \%)$ & $452(87.8 \%)$ \\
\hline & 1 & $53(12.7 \%)$ & $51(9.9 \%)$ \\
\hline & 2 & $16(3.9 \%)$ & $10(1.9 \%)$ \\
\hline & 3 & $1(0.24 \%)$ & $2(0.39 \%)$ \\
\hline \multirow[t]{2}{*}{ Death during follow-up } & Yes & $108(26.0 \%)$ & $167(32.4 \%)$ \\
\hline & No & $308(74.0 \%)$ & $348(67.6 \%)$ \\
\hline
\end{tabular}

${ }^{\text {a }}$ Original (presenting) fracture

${ }^{\mathrm{b}}$ Major fracture included - hip, spine, femur, pelvis or humerus

${ }^{\mathrm{c}}$ Minor fracture included - radius, ulna, hand, tibia, fibula, foot, ribs, patella, clavicle, skull, facial 
patients without a minimal trauma fracture, and those seen in FLS clinic prior to the study period were excluded. Five hundred fifteen patients at the FLS hospital and 416 patients at the non-FLS hospital were included (Fig. 1). The baseline characteristics are shown in Table 1. The FLS hospital recruited a slightly higher, but not significantly different proportion of older patients ( 80 . vs $76.2 \%$ ). When the original fracture type was grouped according to three categories (hip, major, minor), FLS hospital patients had more hip fractures (38.6 vs $22.9 \%$ ), but fewer major, non-hip fractures. When fracture type was grouped according to two categories (hip/major, minor), FLS patients were more likely to have a hip/major fracture as their initial fracture than non-FLS patients (60.8 vs $53.3 \%$ ).

During follow-up, FLS hospital patients were less likely to experience any re-fracture, including both major (8.2 vs $12.0 \%$ ) and minor (4.1 vs $4.8 \%$ ) re-fractures. In models adjusting for original fracture site as a two-level variable (major vs minor), there was a $\sim 30 \%$ reduction in the rate of any re-fracture at the FLS hospital (Table 2). In the final, reduced model, this effect was significant (sub-distribution hazard ratio $(\mathrm{SHR})=0.67$, confidence interval $(\mathrm{CI})$ 0.47-0.95, $p=$ $0.025)$. The cumulative incidence of any re-fracture over the 3 -year follow-up period was $16 \%$ in the non-FLS hospital compared to $\sim 11 \%$ to the FLS hospital (Fig. 1)

Importantly, the effect was particularly evident in the reduction of risk of major re-fractures, with $40 \%$ fewer major subsequent fractures in the FLS hospital. This effect was significant (SHR 0.59, CI 0.39-0.90, $p$ value 0.013) (Table 3). The cumulative incidence function shows a cumulative incidence of major re-fracture of $\sim 10.5 \%$ at non-FLS hospital compared to $\sim 6 \%$ at FLS hospital during study follow-up (Fig. 2). The frequency of minor re-fractures at the FLS hospital was lower than the non-FLS hospital (4.1\% compared with $4.8 \%$ ). This reduction was not as marked as that observed for major re-fractures, and as the frequencies of minor re-fractures at both sites was low, the latter was not analysed as a separate subgroup (Fig. 3).

To assess whether death rates were significantly higher at the FLS hospital after accounting for difference in patient characteristics, we performed an additional analysis. This was a time-to-event analysis in which the outcome was death

Table 2 Proportional hazards regression results for any re-fracture: final (reduced) model (132 re-fracture events)

\begin{tabular}{llc}
\hline Predictor & Sub-distribution HR $(95 \% \mathrm{CI})$ & $p$ value \\
\hline $\begin{array}{l}\text { Presenting hospital: } \\
\quad \text { FLS vs non-FLS hospital }\end{array}$ & $0.67(0.47,0.95)$ & 0.025 \\
$\begin{array}{l}\text { Age group: } \\
\quad \geq 65 \text { vs }<65\end{array}$ & $1.79(1.00,3.18)$ & 0.049 \\
$\begin{array}{l}\text { Original fracture type: } \\
\text { Major vs minor }\end{array}$ & $1.60(1.05,2.44)$ & 0.029 \\
\hline
\end{tabular}

Table 3 Proportional hazards regression results for major re-fracture: reduced model (91 major re-fracture events)

\begin{tabular}{llc}
\hline Predictor & Sub-distribution HR $(95 \% \mathrm{CI})$ & $p$ value \\
\hline $\begin{array}{l}\text { Presenting hospital: } \\
\quad \text { FLS vs non-FLS hospital }\end{array}$ & $0.59(0.39,0.9)$ & \\
$\begin{array}{l}\text { Age group: } \\
\quad \geq 65 \text { vs }<65\end{array}$ & $2.50(1.07,5.84)$ & 0.013 \\
$\begin{array}{l}\text { Gender: } \\
\quad \text { Female vs male }\end{array}$ & $1.83(1.04,3.24)$ & 0.035 \\
$\begin{array}{l}\text { Original fracture type: } \\
\quad \text { Major vs minor }\end{array}$ & $2.31(1.33,4.01)$ & 0.037 \\
\hline
\end{tabular}

prior to re-fracture. The analysis was performed using Cox proportional hazard regression. Variables retained in the final model were "Presenting hospital", "age group", "gender" and "original fracture type". Factors associated with risk of death were age $\geq 65$ (hazard ratio (HR) $=5.2, p<0.001$ ), male gender $(\mathrm{HR}=1.76, p<0.001)$ and major initial fracture $(\mathrm{HR}=2.42$, $p<0.001)$. However, after adjusting for these significant predictors of mortality, there was no significant difference in risk of death at the FLS vs non-FLS hospital $(\mathrm{HR}=1.17, p=0.23$.

\section{Discussion}

We found a significant reduction in risk of any re-fracture of $\sim 30 \%$ (HR 0.67 ; CI $0.47-0.95 ; p 0.025$ ) as well as major refracture of $\sim 40 \%$, (HR 0.59; CI 0.39-0.90; p 0.013) in FLS hospital patients compared to the non-FLS hospital patients. The cumulative incidence of any re-fracture was $\sim 16 \%$ at the non-FLS hospital compared to $\sim 11 \%$ at FLS hospital during study follow-up. The cumulative incidence of major refracture was $\sim 10.5 \%$ at the non-FLS hospital compared to $\sim 6 \%$ at FLS hospital during study follow-up. Older age and

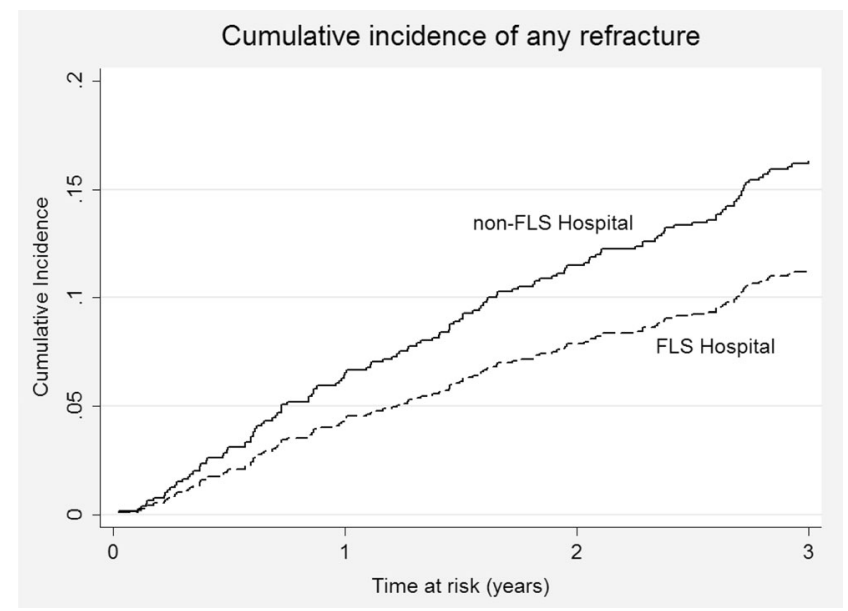

Fig. 2 Cumulative incidence of any re-fracture for the FLS and non-FLS hospitals, adjusted for age group and original fracture type (major/minor) 


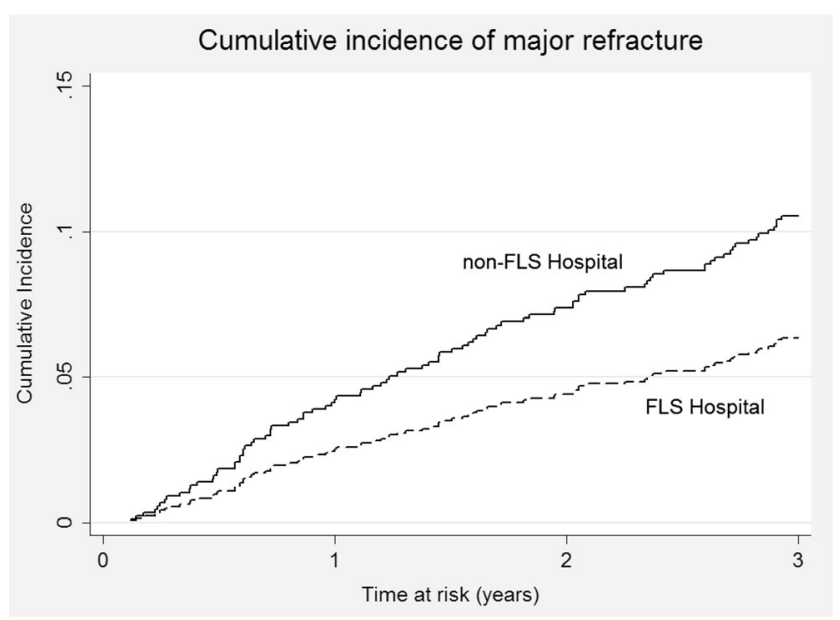

Fig. 3 Cumulative incidence of major re-fracture for the FLS and nonFLS hospitals, adjusted for age group, gender and original fracture type (major/minor)

having a baseline major fracture placed people at greater risk of the next fracture.

Importantly, the $\sim 40 \%$ relative risk reduction in major refractures is in the range of reduction (20-40\%) in nonvertebral fractures reported from placebo-controlled trials of the major anti-osteoporosis medications [29]. The magnitude of the effect of a FLS, however, significantly exceeds the size of effect reported in clinical trials, which typically include patients with osteoporosis but without prior fracture. The number needed to treat (NNT) in these studies is 50-67 to prevent one hip fracture over 3 years [29] compared to the NNT of $20(\sim 5 \%$ absolute reduction in re-fracture rate over 3 years) seen here. It also compares favourably to such widely accepted and applied interventions as statin therapy where the NNT for prevention of one major cardiovascular event in 5 years is 55 [30].

A strength of the study is that it considers the real life effectiveness of a FLS in reducing re-fracture rates, by analysing a large number of incident fracture events in two comparable institutions over defined follow-up periods. Using a competing risk of death in the analysis represents the best methods available to validly analyse observational data. This is one of few studies assessing the improvement in re-fracture rates due to a FLS. The majority of previous studies have focused on issues of implementation and process. While increased investigations and treatment commencements are reported, demonstration that this then translates into fewer re-fractures has generally been lacking $[18,22]$.

Limitations include the retrospective nature of the study. The reliance of hospital sourced data means that patients who re-fractured may have presented to another hospital or health district and may therefore not been identified. While this risk is likely to have been similar across the two sites and unlikely to affect the results, if a bias were to have been present, it is likely to have operated against the FLS hospital, given the nature of its referral base whereby all major trauma is channelled through the one institution, without an adjoining major population centre that could divert some cases.

While the demographic characteristics of the patient population of the two hospitals are similar, further risk factors such as co-morbidities, baseline osteoporotic treatment, vitamin D status and menopausal history were not systematically ascertained during the study and could be possible confounders. Within the limits of this retrospective observational study, a maximal number of potential confounders were collected and included in statistical analysis. The abovementioned issues could only be addressed through a randomised controlled trial, clustered by hospital, but would face serious ethical hurdles given the known risks of osteoporosis and the availability of effective treatment.

The question of which component of a FLS is primarily responsible for the reduction in re-fracture risk is not addressed in this report. We and others have previously reported on the benefit associated with a specialised clinic review [20, 21]. The importance of the current analysis is that it looks at the overall impact of a FLS and encompasses a range of intangibles such as changes in hospital and general practitioner culture.

Within those limitations, the findings utilising intention-totreat approach of analysing all patients over the age of 50 presenting with a MTF show a significant benefit of a FLS.

\section{Conclusion}

There was a $\sim 30 \%$ reduction in the risk of any refracture and a $\sim 40 \%$ reduction in major re-fracture in patients presenting to a hospital with a FLS, when compared to a similar hospital without a FLS. The magnitude of effect of a FLS equates to a NNT of 20, to prevent one re-fracture in 3 years.

Acknowledgments Lydia DeCoster, Dr Narain Kamalaraj, Dr Patrick Pender, Dr Emma Carroll, Dr Fiona Tran, Dr Kathryn Velickovic and Dr Eric Quinn assisted with the data collection.

A/Prof Stephen Oakley and Dr Joyce Lim assisted with interim statistical analysis.

Professor Guy Marks contributed to the initial study design.

A/Prof Kathy Gibson provided liaison and contributed to the discussion of results

Dr Kirtan Ganda and Dr John Van Der Kallen contributed to the discussion of results

Compliance with ethical standards The study was approved by the Hunter New England Human Research Ethics committee.

Conflicts of interest None 
Open Access This article is distributed under the terms of the Creative Commons Attribution-NonCommercial 4.0 International License (http:// creativecommons.org/licenses/by-nc/4.0/), which permits any noncommercial use, distribution, and reproduction in any medium, provided you give appropriate credit to the original author(s) and the source, provide a link to the Creative Commons license, and indicate if changes were made.

\section{References}

1. Cauley JA, Hochberg MC, Lui LY et al (2007) Long-term risk of incident vertebral fractures. JAMA 298(23):2761-7

2. Center JR, Bliuc D, Nguyen TV et al (2007) Risk of subsequent fracture after low-trauma fracture in men and women. JAMA 297(4):387-94

3. Hodsman AB, Leslie WD, Tsang JF et al (2008) 10-year probability of recurrent fractures following wrist and other osteoporotic fractures in a large clinical cohort: an analysis from the Manitoba Bone Density Program. Arch Intern Med 168(20):2261-7

4. Kanis JA, Johnell O, De Laet C et al (2004) A meta-analysis of previous fracture and subsequent fracture risk. Bone 35(2):375-82

5. Klotzbuecher CM, Ross PD, Landsman PB et al (2000) Patients with prior fractures have an increased risk of future fractures: a summary of the literature and statistical synthesis. J Bone Miner Res 15(4):721-39

6. Edwards BJ, Bunta AD, Simonelli C et al (2007) Prior fractures are common in patients with subsequent hip fractures. Clin Orthop Relat Res 461:226-30

7. Cooper C, Atkinson EJ, Jacobsen SJ et al (1993) Population-based study of survival after osteoporotic fractures. Am J Epidemiol 1993(137): 9

8. Leibson CL, Tosteson AN, Gabriel SE et al (2002) Mortality, disability, and nursing home use for persons with and without hip fracture: a population-based study. J Am Geriatr Soc 50(10):1644-50

9. Magaziner J, Simosick EM, Kashner TM et al (1990) Predictors of functional recovery one year following hospital discharge for hip fracture: a prospective study. J Gerontol 45(3):M101-7

10. Brauer CA, Coca-Perraillon M, Cutler DM et al (2009) Incidence and mortality of hip fractures in the United States. JAMA 302(14):1573-9

11. Gullberg B, Johnell O, Kanis J (1997) World-wide projections for hip fracture. Osteoporos Int 7(5):407-13

12. Eisman JA, Bogoch ER, Dell R et al (2012) Making the first fracture the last fracture: ASBMR task force report on secondary fracture prevention. J Bone Miner Res 27(10):2039-46

13. Akesson K, Marsh D, Mitchell PJ et al (2013) Capture the fracture: a best practice framework and global campaign to break the fragility fracture cycle. Osteoporos Int 24(8):2135-52

14. Marsh D, Akesson K, Beaton DE et al (2011) Coordinator-based systems for secondary prevention in fragility fracture patients. Osteoporos Int 22(7):2051-65
15. Adachi JD, Kennedy CC, Papaloannou A et al (2009) Treating osteoporosis in Canada: what clinical efficacy data should be considered by decision makers? Osteoporos Int 20(10):1785-93

16. Ganda K, Puech M, Chen JS et al (2013) Models of care for the secondary prevention of osteoporotic fractures: a systematic review and meta-analysis. Osteoporos Int 24(2):393-406

17. McLellan AR, Wolowacz SE, Zimovetz EA et al (2011) Fracture liaison services for the evaluation and management of patients with osteoporotic fracture: a cost effectiveness evaluation based on data collected over 8 years of service provision. Osteoporos Int 22(7): 2083-98

18. Sale JE, Beaton D, Posen J et al (2011) Systematic review on interventions to improve osteoporosis investigation and treatment in fragility fracture patients. Osteoporos Int 22(7):2067-82

19. Huntjens KM, van Geel TA, van den Bergh JP et al (2014) Fracture liaison service: impact on subsequent nonvertebral fracture incidence and mortality. J Bone Joint Surg Am 96(4):e29

20. Lih A, Nandapalan H, Kim M et al (2011) Targeted intervention reduces refracture rates in patients with incident non-vertebral osteoporotic fractures: a 4-year prospective controlled study. Osteoporos Int 22(3):849-58

21. Van der Kallen J, Giles M, Cooper K et al (2014) A fracture prevention service reduces further fractures two years after incident minimal trauma fracture. Int J Rheum Dis 17(2):195-203

22. Astrand J, Nilsson J, Thorngren K (2012) Screening for osteoporosis reduced new fracture incidence by almost half: a 6-year followup of 592 fracture patients from an osteoporosis screening program. Acta Orthop 83(6):661-5

23. Sanders KM, Nicholson GC, Watts JJ et al (2006) Half the burden of fragility fractures in the community occur in women without osteoporosis. When is fracture prevention cost-effective? Bone 38(5):694-700

24. Alonso-Coello P, García-Franco AL, Guyatt G et al (2008) Drugs for pre-osteoporosis: prevention or disease mongering? BMJ 336(7636):126-9

25. Järvinen TL, Michaëlsson K, Jokihaara J et al (2015) Overdiagnosis of bone fragility in the quest to prevent hip fracture. BMJ. doi:10. 1136/bmj.h2088

26. Fine JP, Gray R (1999) A proportional hazards model for the subdistribution of a competing risk. J Am Stat Assoc 94:496-509

27. Wolkewitz M, Cooper BS, Bonten MJ et al (2014) Interpreting and comparing risks in the presence of competing events. BMJ 349: g5060. doi:10.1136/bmj.g5060

28. Australian Bureau of Statistics (2011) Census of population and housing: socio-economic indexes for areas (SEIFA), Australia 2011. ABS, Canberra

29. Crandall CJ, Newberry SJ, Diamant A et al (2014) Comparative effectiveness of pharmacologic treatments to prevent fractures: an updated systematic review. Ann Intern Med 161(10):711-23

30. Taylor F, Huffman MD, Macedo AF, et al (2013) Statins for the primary prevention of cardiovascular disease. Cochrane Database Syst Rev (1):CD004816. doi:10.1002/14651858 